\title{
Hysterosalpingography: A Re-Emerging Study in Health Care Transformation
}

\author{
JosephBako Igashi ${ }^{1}$,DlamaZira Joseph ${ }^{2}$,Gloria Joseph ${ }^{2}$,Saidu Kadas ${ }^{2}$, \\ GeofreyLuntsi $^{3}$, Flavious Nkubli ${ }^{3}$, Samuel Laushugno ${ }^{4}$, ObettaChinedu T. ${ }^{4}$ \\ 1.Radiology Department Ahmadu Bellouniversity Teaching Hospital Zaria, Nigeria. \\ 2.AbubakarTafawaBalewa University Teaching Hospital Bauchi, Nigeria \\ 3.Department of Medical Radiography University of Maiduguri,Bornostate,Nigeria \\ 4.Department of Radiography and Radiological Sciences, NnamdiAzikiwe University Awka, Nigeria \\ ${ }^{1}$ Corresponding Author: Joseph BakoIgashi.MBBS,FWACS \\ EMAIL: Josephbakoigashi@yahoo.co.uk
}

\begin{abstract}
:
Background: Hysterosalpingography is a commonly performed examination due to recent advances and improvement in reproductive medicine.It involves the radiographic delineation of female reproductive organs. The major role of Hysterosalpingography is to investigate the uterus and the fallopian tubes which is achieved following the introduction of contrast agent into the female reproductive system.

Objectives: The objective of this study is to analyze the common pathological requests and findings of Hysterosalpingographypatients in AhmaduBello University Teaching Hospital Zaria.

Methodology: Two hundred and thirty five (235) requestcards, reports/films from Radiology Department of Ahmadu Bello University Teaching Hospital Zaria were reviewed retrospectively and analyzed in order to determine common indications, provisional diagnosis, age of patients undergoingHysterosalpingographyprocedure. The result was obtained and categorized according to their clinical findings and they were analyzed using Statistical package for social science Version 16.0.

Results: Results showed $8.9 \%(n=25)$ Cervicalabnormalities, uterine abnormalities accounted for $25 \%(n=$ 67), abnormalities of the fallopian tubes $44.1 \%(n=124)$ while peritoneal adhesions were $19.57 \%(n$ $=55)$. Most of the referrals were those who had secondary infertility $40.4 \%(n=95)$ as opposed to those with primary infertility $29.4 \%(n=69)$

Conclusion:Hysterosalpingography is effective in evaluating the internal organs of female genital tract in clinically suspected cases of infertility. It is a re-emerging study in reproductive medicine and valuable in health caretransformation.
\end{abstract}

Key Words: Hysterosalpingography, findings, contrast agent, infertility, pathological.

\subsection{Background}

\section{Introduction}

Hysterosalpingography is a fluoroscopically guided contrast examination of the female genital tract using about 10 to 20 mills of contrast media usually urografin in our environment ${ }^{[1]}$. HSG evaluates the integrity of the female genital tract in clinically suspected cases of infertility. It is valuable in radiographic delineation of uterine and tubal cavities and it also serve as a vital tool for the diagnostic evaluation of conjugal infertility ${ }^{[2],[3]}$. HSG procedure offers the best method to evaluate the female reproductive tract ${ }^{[4],[8]}$.

Infertility is defined as inability of a couple to establish pregnancy within a certain period of regular unprotected coital exposure usually one year ${ }^{4}$. It is primary, if the couple has had no previous pregnancy irrespective of the outcome of the pregnancy ${ }^{[3],[4],[10]}$.In radiographic practice however, the number of HSG procedures have increased dramatically over the past few years, the increase is likely due to advances in reproductive medicine and the tendency of women delaying child bearing ${ }^{[8]}$

HSG is considered as gold standard for assessment of fallopian tubes giving relevant information and vital clues about patency and morphology ${ }^{[6],[7]}$. It remains a very important diagnostic tool in the investigation of the infertile women especially in Nigeria ${ }^{[16]}$. The advantages of HSG include producing a global image of the reproductive canal with details of endothelial architecture from cervix to fimbriae using water soluble contrast media ${ }^{[8]}$.HSG is the most frequently used diagnostic tool to evaluate the endometrial cavity. However, meticulous and well executed procedure performed provides accurate information about endocervical canal,viz a viz diameter and configuration of the internal canal, location and direction of fallopian tubes, status of the fimbriae and spillage into the peritoneal cavity. HSG must be carefully interpreted as under or over interpretation may be misleading in the management of patient ${ }^{[4],[12]}$. 
Recent advances in reproductive medicine have generated a demand for more accurate imaging methods for identifying the specific cause of infertility and other gynecological disorders ${ }^{[8],[10]}$. Hysterosonography and ultrasonography are valuable in the evaluation of the endometrium while Magnetic Resonance Imaging is useful in evaluating the ovaries and uterine myometrium for uterine contour myomas. Multidetector Computed Tomography is capable of depicting both the external and internal surfaces of the uterus, fallopian tubes and other pelvic organs providing high resolution dat ${ }^{[12],[13],[15] \text {. }}$

Infertility is the commonest complain encountered in the gynecology outpatient clinics in Nigeria $[11],[12],[15]$. Infertility is common worldwide, it has been estimated that about one in every ten couples has difficulty in conceiving ${ }^{[10],[12]}$. Majority of patients that come to gynecological out patients clinic in Ahmadu Bello University Teaching Hospital Zaria complained of infertility which are subsequently referred to radiology department for HSG. The frequency of this referral to the Radiology department in this teaching hospital was the major problem that necessitated this research.

This study is aimed at determining the common pathological findings in HSG patients at radiology department of ABUTH Zaria.

\section{Methodology}

This research work is a three and half year retrospective study carried out at the Radiology department of Ahmadu Bello University teaching hospital Zaria. A total of two hundred and thirty five(235) studies of women between 16 to 45 years were referred to the department.After due consent From the hospital ethical committee, Information such as clinical indications/ provisional diagnosis, age ofpatients and findings were extractedfrom the records, films of inappropriate reports were reviewed by certified and experienced Radiologist. This is to ensure accuracy of research data. Thedata was analyzed using SPSS version 16.0.

\subsection{Result}

\section{Data Analysis And Presentation}

The result shows that the commonest age group affected were between the ages of 20 years and 39 years $84.8 \%(\mathrm{n}=198)$ with peak range at $25-29$ years $32.0 \%(\mathrm{n}=75)$. There was only $1(0.4 \%)$ patient below 20 years . the ages of $18(7.5 \%)$ patients have incomplete/insufficient clinical details.

Table I : Shows the age distribution of patients $(\mathrm{n}=235)$

\begin{tabular}{|l|l|l|}
\hline Ages (year) & No of patients & Percentage $\%$ \\
\hline$\leq 20$ & 1 & 0.4 \\
\hline $20-24$ & 27 & 11.5 \\
\hline $25-29$ & 75 & 32.0 \\
\hline $30-34$ & 45 & 19.1 \\
\hline $35-39$ & 51 & 22.2 \\
\hline $40-45$ & 18 & 7.5 \\
\hline Unspecified & 18 & 7.5 \\
\hline Total & $\mathbf{2 3 5}$ & $\mathbf{1 0 0}$ \\
\hline
\end{tabular}

Table II: This table shows clinical indications requiring HSG investigation

\begin{tabular}{|l|l|l|}
\hline Clinical indication & Number (n) & Percentage \% \\
\hline Primary Infertility & 69 & 29.4 \\
\hline Secondary infertility & 95 & 40.4 \\
\hline Recurrent Abortion & 10 & 4.3 \\
\hline Sub-fertility & 3 & 1.3 \\
\hline Secondary Amenorrhea & 10 & 4.3 \\
\hline Post myomectomy & 4 & 1.7 \\
\hline Uterine Fibroid & 43 & 18.2 \\
\hline Cervical incompetence & 1 & 0.4 \\
\hline Total & $\mathbf{2 3 5}$ & $\mathbf{1 0 0}$ \\
\hline
\end{tabular}

The above table II Presents higher percentage of secondary infertility to primary infertility, which shows $29.4 \%(n=69)$ of women with primary infertility, while $40.4 \%(n=95)$ were with secondary infertility only $0.4 \%(\mathrm{n}=1)$ patient had cervical incompetence.

Table III:Distribution of findings from HSG reports $(\mathrm{n}=235)$

\begin{tabular}{|c|c|c|c|}
\hline \multicolumn{2}{|c|}{ FINDINGS } & NUMBERS OF CASES & PERCENTAGE (\%) \\
\hline 1) & Normal & 10 & 3.56 \\
\hline 2) & CERVIX & & \\
\hline a) & Synechiae & 14 & 4.98 \\
\hline b) & Incompetence & 1 & 0.36 \\
\hline c) & Fibroid & 10 & 3.56 \\
\hline
\end{tabular}


Hysterosalpingography: A Re-Emerging Study in Health Care Transformation

\begin{tabular}{|c|l|l|}
\hline TOTAL & $\mathbf{2 5}$ & $\mathbf{8 . 9}$ \\
\hline 3) UTERUS & & \\
\hline a) Asher man's syndrome/ synechiae & 21 & 7.47 \\
\hline b) Fibroid & 45 & 16.0 \\
\hline c) Congenital anomaly & 1 & 0.36 \\
\hline TOTAL & $\mathbf{6 7}$ & $\mathbf{2 3 . 8 4}$ \\
\hline 4) $\quad$ & \\
\hline a) TuLLOPIAN TUBES & 62 & 22.1 \\
\hline i) Unilateral & 34 & 12.0 \\
\hline ii) Bilateral & 28 & 9.96 \\
\hline b) Hydrosalpinges & 62 & 22.1 \\
\hline i) Unilateral & 32 & 11.4 \\
\hline TOTAL Bilateral $\quad 30$ & 10.7 \\
\hline 5) PERITONEAL ADHESIONS & $\mathbf{1 2 4}$ & $\mathbf{4 4 . 1}$ \\
\hline a) Pelvic adhesions & 23 & \\
\hline b) Perifimbrial adhesions & 32 & 8.19 \\
\hline TOTAL tubes & $\mathbf{5 5}$ & 11.39 \\
\hline \multicolumn{2}{|l|}{} & $\mathbf{1 9 . 5 7}$ \\
\hline SUM TOTAL & $\mathbf{2 8 1}$ & \\
\hline
\end{tabular}

\section{Discussions}

The recommended period to perform HSG is towards the end of the first week after the Menstrual period when the Isthmus is at its most distensible and the fallopian tubes are most readily filled by contrast medium. HSG is avoided in the second half of the cycle because of fear of inadvertently irradiating an ongoing pregnancy and because the thickened Endometrium which could lead to false positive diagnosis of cornual occlusion $[101,[12],[19]$

The study showed that the commonest age group affected was between the ages of 20 years and 39 years $198(84.8 \%)$ with peak range at $25-29,75(32.0 \%)$. This is so because the active reproduction period in females is this age range. The ages of $18(7.5 \%)$ patients were not specified while $1(0.4 \%)$ was below 20 years. This findings concurs with previous studies ${ }^{[1],[4]}$. Routine (HSG) is a gold standard for evaluating the structural changes in the uterus and fallopian tubes ${ }^{[6],[7]}$.In this finding, it was found that $29.4 \%$ (69) of the investigated women had primary infertility while $40.4 \%$ (95) were with secondary infertility. Previous studies showed varying values for primary and secondary infertility ${ }^{[1],[8]}$.

There was higher percentage of secondary infertility compared to primary infertility in previousresearches and this is in harmony with the findings in this research work ${ }^{[1],[4],[5],[8]}$. This shows that the main clinical problem requiring HSG is secondary infertility ${ }^{[1],[4],[5],[8]}$.From the findings in this work it was noted that some of the patients presented more than one pathological condition. Five 10 (3.56\%) cases in this study demonstrated normal findings while $230(97.5 \%)$ revealed abnormal findings with a breakdownof tubal, uterine, cervical and peritoneal factors of $92(39.1 \%), 65(27.7 \%), 13(5.6 \%)$ and $60(25.5 \%)$ respectively. This work agrees with prevailing findings and suggests that there are many cases of infertility with Tubal factors as the main cause. Research shows that this may be secondary to disease such as peritonitis, endometriosis and pelvic inflammatory diseases ${ }^{[4],[8] .}$

Majority of patients have unilateral tubal occlusion 34(12.0\%) this disagree with the earlier findings in Port-Harcourt (16.6\%) and $(17.5 \%)^{[5]}$. In their study they assessed the value of (HSG) in tubal patency and peritubal adhesion, they found tubal factors in $89.2 \%$, Uterine $9.4 \%$ peritoneal $12.7 \%$ when compared with this study finding of $124(44.1 \%)$ tubal factors, $67(23.84 \%)$ uterine factors and $55(19.57 \%)$ peritoneal factors ${ }^{[6],[8],[12]}$. This finding shows that tubal abnormality is most likely due to infection which may be sub-clinical in many cases and especially sexually transmitted disease. The tubal abnormality may be explained by possible spread of infection from chronically inflamed appendix to the tube. The appendicular inflammation may however be subclinical in most cases it is as a result of antibodies in this environment ${ }^{[8],[10],[14]}$.

Uterine fibroid $45(16.0 \%)$ is the commonest uterine abnormity in this study which concur with that of Enugu ${ }^{[5]}$. Leiomyomas generally referred to as fibroids are benigntumors arising from smooth muscles of the uterus and fallopian tubes ${ }^{[20]}$. The exact etiology of fibroid is uncertain, but it is diagnosed in $20 \%$ to $25 \%$ of women 35years of age or older incidence is up to 7times higher in black women compared to Caucasians[ ${ }^{8],[20]}$. Evidence strongly suggests that fibroids are estrogen depended and diminish with age hence their rarity in postmenopausal and prepubertalage ${ }^{[20]}$. Fibroids are generally classified according to their location ${ }^{[20]}$.Such classification results in fibroids being described as intramural, submucosal, subserosaland cervical. A fibroid may also be pedunculated,If it develops some pedicle which attaches itself to the uterine wall and grows externally into the peritoneal cavity ${ }^{[15],[18],[20]}$

Pelvic adhesions $23(8.19 \%)$, perifimbrial adhesions 32(11.4\%) were also seen among the peritoneal abnormalities. HSG is very weak in diagnosis of these abnormalities. However, it could sometime be inferred by 
delaying or localized spill, lack of tubal mobility, abnormality and tubal contour. The extrinsic tubal pathology is better evaluated with Laparoscopy although HSG is inferior to laparoscopy in defining peritubal disease, this agrees with mostfindings ${ }^{[2],[3] .}$

Bilateral hydrosalpinges 30(10.7\%) and unilateral 32(11.4\%) varies with the study carried out in Enugu ${ }^{[8]}$. Hydrosalpinx is enlongated tortuous dilatation of the Ampullary portion of the fallopian tube that shows contrast retention even in delayed film. The incidence of bilateral tubal occlusion $28(9.96 \%)$ and unilateral hydrosalpinx $32(11.4 \%)$ reveals that infection is not the leading etiology factor in infertility in our environment. This is in disagreement with the findings of Enugu ${ }^{[8]}$.Prevention of sexually transmitted disease and its prompt treatment will decrease the incidence of tubal blockage since they are the causes of tubal blockage and hydrosalpinges, since they are the end result of acute and chronic salpingitis. It must be noted that the evaluation of tubal patency could be misinterpreted many times due to some technical difficulties resulting from tubal spasm or under filling. The usual practice is to give antispasmodium drug (20mg Buscopan) to patients suspected to have tubal spasm.

There was only one 1(0.36) case of cervical incompetence. Secondary infertility is common points to pelvic infection complicating mismanaged pregnancies, septic abortions and sexually transmitted diseases.

Since most of the case of tubal occlusion and the uterine and cervical synechiae are secondary to poorly treatedinfection, it is strongly advised that good hygiene and proper treatment of any form of infection be advocated.

\section{Conclusion}

The commonest clinical problem requiring investigation on HSG patients at ABUTH Zaria is uterine fibroid. Only one case ofcongenital anomalyis noted. Despite the fact that secondary infertility is common points to uterine fibroid, a study to establish associated factors is recommended.

Also, since most cases of Ashermer's syndrome, tubal occlusion and peritoneal adhesions are secondary to poorly treated infection.it is strongly advised that good hygiene and proper treatment of any form of infection be advocated. HSG is vital in modern gynecological practice.

\section{References}

[1]. Agwuna K.K. Anyawu G.E. (2006). Anatomical defects at HSG in Patients with infertility. Journal of experimental and clinical Anatomy; 5(2):7-12.

[2]. Akano A.O, Olatunji O.O, Agaja E.O., and Olakoju P.E. (200). Servers Reaction after buscopan premedication for HSG. Archives of Nigerian medicine and medical sciences; 1(1): 23-25

[3]. Arsian S.A, Itabeys, Koc C, Var T, Bilge U (2004). Evaluation of infertile Women; HSG, Office hysteroscopy or hysterography; which is Better? Journal of fertility; 4:1-4

[4]. Asaleye C.M., Adetiloye V.A., Oyinlola T.O. (2004) Review of HSG reports done for fertility. Nigerian journal of health sciences; 4:36-39.

[5]. Dhaliwal L.K, Gupta K.R. Aggarwal N. (1999) is HSG an important tool in modern gynaecological practice. International journal of fertile women 44:14-16.

[6]. Ezegwui H.U., Mgbor S.O, Ikeme A.C, (2001). Patients journal of obstetrics and gynaecology: 18:14-16.

[7]. Ghazi A, Saddique M.S., Saddique N, Jabbar S. (2007), in tertiary care Hospital. Parkistan journal of surgery; 23:283-286.

[8]. Mgbor S.O. Patten of Hysterosalpingography (HSG) findings in Gynaecology patients in Enugu, Nigeria journal; 47:14-16.

[9]. Kawuwa M.B, Idrisa A, Kyari O, Habu S.A, Adebayo A. (2005). Gonadotropin level among women in Maiduguri: Borno medical journal; 2:13-16.

[10]. Kawuwa M.B, Maigari A.G, Audu B.M, (2007). Indications and implications of hysterectomy in Maiduguri; Kanem journal of medical science; 1(1): 20-25.

[11]. Onah H.E, Mgbor S.O, U.A (2006). Out come of tubal surgeries in University of Nigeria Teaching Hospital Enugu, Nigeria journal of clinical practice; $9(1)$ : 44-47.

[12]. Okogbenin S.A, Okepere E.E, Eigbefoh J.I, Ogbo F.O, Okonta P.I, Onuh S.O. (20060. Gynaecological indication for abdominal hysterectomy in the university of Benin teaching hospital. Archieves of clinical journal, 1:56-63.

[13]. Research papers on infertility and reproductive medicine. Proceeding of $18^{\text {th }}$ world congress on infertility and sterility (IFS). 2004); 1271: 11-14.

[14]. Shanmugasundaram R., Ganesh, GopalakrishnaaKakre N.S (20080 Youssef's syndrome is there a better way to diagnose? 24:26970

[15]. Trancers M.L, (1986). A review of obstetrics and gynaecological survey. Vesicouterine fistula; 41:743-53.

[16]. Umdagas H.A, Kawuwa M.B, Hajara U.S, Mohammed S. (2006), Hsgand infertilityJournal of obstetrics and gynaecology; 26 (4):351-2.

[17]. Ugwu A.C, Imo A.O, Erondo O.F (2008). Impacts of demographic and Psychosocial factors on hysterosalpingography pain and comfort; Journal of Radiography (UK) accepted.

[18]. Ronald G.G. The female reproductive system in Grainger RG, Alisa DJ, editors. Diagnostic radiology, Vol.3 Philadelphia: Churchill living store: $1993 ; 40: 139-59$.

[19]. Crofton M. Gynaecology imaging. Textbook of Radiology and Imaging $7^{\text {th }}$ ed. Vol.2. 2007 plot.

[20]. Incidence and age distribution of uterine fibroids at FMC owerri, Nigeria. Journal of medical imaging and Radiationtherapy. Vol1 No.3.Nov.2012. 25-29. 